\title{
Cure-Dependent Viscoelastic Analysis on the Residual Stresses and Distortion Created in Composite Corner During Curing
}

\author{
Jiang-Tao ZHANG ${ }^{a, *}$, Yun-Dong SHANG ${ }^{b}$, Mei ZHANG ${ }^{C}$, Li-Sheng LIU ${ }^{d}$, \\ Peng-Cheng $\mathrm{ZHAl}^{\mathrm{e}}$ and Shu-Xin $\mathrm{LI}^{\mathrm{f}}$
}

\author{
Department of Engineering Structure and Mechanics, School of Science \\ Wuhan University of Technology, Wuhan 430070, PR China \\ azhjiangtao@whut.edu.cn, ${ }^{\text {b7} 75038280 @ q q . c o m, ~}{ }^{\text {cz } z h a n g m e i @ w h u t . e d u . c n, ~}{ }^{d}$ liulish@whut.edu.cn, e \\ pczhai@126.com, shuxinli01@163.com \\ * Corresponding author
}

Keywords: Polymer-matrix composites(PMCs), Curing, Residual stress, Distortion, Finite element analysis(FEA)

\begin{abstract}
A cure-dependent viscoelastic constitutive model is simply derived, and verified by comparing the results predicted by the derived model with the theoretic solution of a rod model and the standard ABAQUS elastic solution of a three-dimensional (3D) cross-ply laminate [0/90] s. A 3D simulation of an AS4/3501-6 composite corner is performed to predict the evolution of the residual stresses and distortion during the entire cure process, and the effect of boundary condition is investigated. The results show that the model for the predictions of the residual stresses and distortion in composite component should include both the curing process and cooling process, and the effects of stress relaxation, chemical shrinkage and thermal deformation should be considered. A proper boundary condition is important for the accuracy of the numerical simulations.
\end{abstract}

\section{Introduction}

The accumulation of residual stress in polymer matrix composites (PMCs) during curing is inevitable due to their inherent anisotropy and inhomogeneity, and it can deteriorate the performance of composite structures. The cure-dependent constitutive model is essential for the prediction accuracy, and it should be able to capture the main mechanisms relevant to the residual stress and shape distortion of PMCs. The earliest analysis of residual stresses in PMCs is based on the assumption that PMCs are in the stress-free state at the high cure temperature, residual stresses only develop during the cooling process[1, 2]. These models are questionable for several reasons, such as the chemical shrinkage and residual stress developed before cool-down are not taken into account[3-6]. To capture the effects of these factors, various cure-dependent elastic models were proposed in the last two decades, such as the path dependent constitutive model [4,5] and the cure-hardening, instantaneous linear elastic(CHILE) model[7]. Abouhamzehan et al [8] developed an analytical model to predict the warpage and residual stresses developed during curing of Fibre Metal Laminates (FMLs) based on the Classical Laminate Theory (CLT). In these models, however, stress relaxation during the cure process is still not considered.

In recent years, the cure-dependent viscoelastic models are widely used to simulate the development of residual stresses in PMCs. The viscoelastic models are proposed based on the recognition that the polymer matrix of PMCs changes from liquid-like material in the early stages of cure to a viscoelastic solid at the end[9,10]. White and Hahn [11,12] firstly developed a process-dependent viscoelastic model for the investigation of residual stress in composite laminates during the entire cure cycle and validated the model by the intermittent cure of unsymmetric cross-ply laminates in which processing induced curvatures were measured. White and Kim [3] and Lee and Kang[13] introduced thermo-viscoelastic constitutive equations depending on degree of cure and temperature, and investigated the residual stresses of Hercules AS4/3501-6 composite during the entire cure cycle by executing their models into a 2D finite element model (FEM). More recently, the thermo-viscoelastic analysis on the development of residual stresses in complex 
composite structures using the 3D FEA were frequently reported in the literatures[14-18]. Ding et al [18] compared the predictions of the cure-dependent thermo-viscoelastic, path-dependent and CHILE constitutive laws. These works revealed that the thermo-viscoelastic analyses throughout the entire cure cycle could predict the development of residual stresses in composite structure in a more realistic manner.

The present work simply derived a cure-dependent viscoelastic model at first. Then using the viscoelastic data and reaction kinetics provided by White and Kim [3,20,21], the viscoelastic model was verified by comparing the predicted results with those predicted by the theoretical result of a rod model and a standard ABAQUS elastic model of a plate model. The highlight of this work is that the cure-dependent constitutive model was applied to a composite corner to investigate the effects of boundary condition on the development of residual stresses and shape distortion during curing, and the effect of stress relaxation, chemical shrinkage and thermal contraction is analyzed.

\section{Viscoelastic Constitutive Equations}

If the composite material is assumed to be thermorheologically simple at a constant degree of cure $\alpha$, based on the time-temperature superposition principle, the viscoelastic constitutive equation for relaxation of residual stresses generated in composites during curing can be expressed by the following hereditary integral[3,13]

$$
\begin{aligned}
& \sigma_{i}(t)=\int_{0}^{\xi} C_{i j}\left(\alpha, T_{r}, \xi-\xi^{\prime}\right) \frac{\partial \varepsilon_{j}\left(\xi^{\prime}\right)}{\partial \xi^{\prime}} d \xi^{\prime} \\
& \varepsilon_{j}(t)=\widetilde{\varepsilon}_{j}(t)-\beta_{j} \Delta T-\eta_{j} \Delta \alpha
\end{aligned}
$$

Where $\sigma$ and $\varepsilon$ represent the stress and mechanical strain, $\tilde{\varepsilon}$ is the total strain, $C_{i j}$ represents the time-dependent stiffness matrix, $\beta$ and $\eta$ represent coefficients of thermal expansion (CTE) and coefficients of chemical shrinkage (CCE), respectively. $\Delta T$ and $\Delta \alpha$ indicate the changes in temperature and degree of cure. $t$ is the current time, $\tau$ is a dummy variable for integration and indicates the past time. $T_{r}$ is the reference temperature. $\xi$ and $\xi^{\prime}$ are the reduced times and can be calculated as follows:

$$
\xi=\xi(t)=\int_{0}^{t} \frac{1}{a_{T}(\alpha, T(s))} d s \text { and } \xi^{\prime}=\xi(\tau)=\int_{0}^{\tau} \frac{1}{a_{T}(\alpha, T(s))} d s
$$

Where $a_{T}$ is the shift factor and is functionally dependent on $\alpha$ and $T$. In Eq (1), $C_{i j}\left(\alpha, T_{r}, \xi-\xi^{\prime}\right)$ is noted as $C_{i j}\left(\alpha, \xi-\xi^{\prime}\right)$ and be approximated by Prony series as[3]:

$$
C_{i j}(\alpha, \xi)=C_{i j_{\infty}}+\sum_{m=1}^{M} C_{i j_{m}} \exp \left(\frac{-\xi}{\rho_{m}(\alpha)}\right)
$$

Where $C_{i j_{\infty}}, C_{i j_{m}}$ and $\rho_{m}(\alpha)$ are the fully relaxed stiffnesses, the discrete stiffnesses and the cure-dependent discrete relaxation times for the $m^{\text {th }}$ elements respectively. Substituting Eq. (4) into Eq. (1), the viscoelastic constitutive equation becomes

$$
\sigma_{i}(t)=C_{i j_{\infty}} \varepsilon_{j}(t)+\sum_{m=1}^{M} C_{i j_{m}} \int_{0}^{\xi_{t}} \exp \left(-\frac{\xi_{t}-\xi^{\prime}}{\rho_{m}(\alpha)}\right) \frac{\partial \varepsilon_{j}\left(\xi^{\prime}\right)}{\partial \xi^{\prime}} d \xi^{\prime}
$$

In order to solve the integral in Eq. (5), the recursive algorithm first proposed for linear viscoelastic integrals by Tayer et al[19] is applied in this work. The integral in Eq. (5) is referred to as $q_{j m}(t)$. Assuming a sufficiently small time increment $\Delta t$, the integral can be rewritten as

$$
q_{j m}(t)=\int_{0}^{\xi_{t-\Delta t}} \exp \left(-\frac{\xi_{t}-\xi^{\prime}}{\rho_{m}(\alpha)}\right) \frac{\partial \varepsilon_{j}\left(\xi^{\prime}\right)}{\partial \xi^{\prime}} d \xi^{\prime}+\int_{\xi_{t-\Delta t}}^{\xi_{t}} \exp \left(-\frac{\xi_{t}-\xi^{\prime}}{\rho_{m}(\alpha)}\right) \frac{\partial \varepsilon_{j}\left(\xi^{\prime}\right)}{\partial \xi^{\prime}} d \xi^{\prime}
$$

Where $\xi_{t}=\xi_{t-\Delta t}+\Delta \xi_{t}$ and $\Delta \xi_{t}=\int_{t-\Delta t}^{t} \frac{1}{a_{T}(\alpha, T(s))} d s$.

In order to solve Eq. (6), it is assumed that the degree of cure and temperature are constant during 
the small time step $\Delta t$, and $\varepsilon_{j}\left(\xi^{\prime}\right)$ is a linear function of the reduced time $\xi^{\prime}$. Then the integral of Eq. (6) can be solved as a recursive formulation and given by

$$
q_{j m}(t)=\exp \left(-\frac{\Delta \xi_{t}}{\rho_{m}\left(\alpha_{t}\right)}\right) q_{j m}(t-\Delta t)+\frac{\rho_{m}\left(\alpha_{t}\right)}{\Delta \xi_{t}}\left(1-\exp \left(-\frac{\Delta \xi_{t}}{\rho_{m}\left(\alpha_{t}\right)}\right)\right) \Delta \varepsilon_{j}^{t}
$$

Substituting Eq.(7) into Eq.(5), the current stress is finally expressed as

$$
\sigma_{i}(t)=C_{i j_{\infty}} \varepsilon_{j}(t)+\sum_{m=1}^{M} C_{i j_{m}}\left(\exp \left(-\frac{\Delta \xi_{t}}{\rho_{m}\left(\alpha_{t}\right)}\right) q_{j m}(t-\Delta t)+\frac{\rho_{m}\left(\alpha_{t}\right)}{\Delta \xi_{t}}\left(1-\exp \left(-\frac{\Delta \xi_{t}}{\rho_{m}\left(\alpha_{t}\right)}\right)\right) \Delta \varepsilon_{j}^{t}\right)
$$

In Eq. (8), the first part represent the contribution of the fully relaxed stiffnesses to the residual stress, and the corresponding stress cannot be relaxed during the cure process. The second part represent the contribution of the discrete stiffnesses, it is time-dependent and stress relaxation can happen for this part.

\section{Verification on the Constitutive Model}

In this work, the behavior of the Hercules AS4/3501-6 composite is used as the example. The cure-dependent viscoelasticity and reaction kinetics of AS4/3501-6 composite were systematically measured and reported by Kim and White[3, 20, 21]. The viscoelastic constitutive equation Eq. (8) is implemented in the ABAQUS finite element code through the user subroutine UMAT. A user subroutine UEXPAN in ABAQUS was written to consider the thermal strains and the chemical shrinkage strains of AS4/3501-6 composite during the cure process. ABAQUS is widely used in the thermo-viscoelastic analysis of composites during cure due to its powerful nonlinear and multi-field couple analysis capability, and various and friendly user interfaces[17, 18]. All of the parameters in the constitutive equation and reaction kinetics equations are from the references[3,20,21].

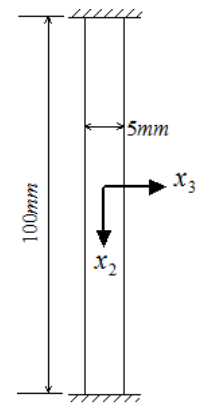

(a)

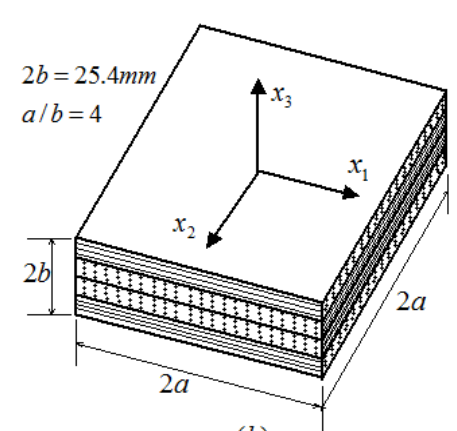

(b)

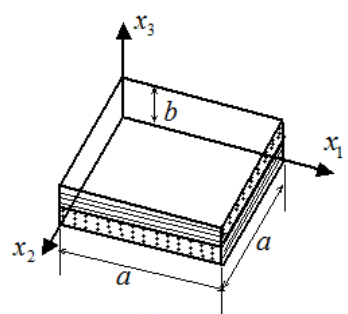

(c)

Figure 1. Geometry models used for verification (a) one-dimensional rod with constrained ends, (b) three-dimensional laminate $[0 / 90]_{\mathrm{s}}$ and (c) $1 / 8^{\text {th }}$ model used to represent model (b)

Two finite element simulations were carried out as shown in Fig. 1. First a rod with its ends constrained was examined. The second model simulated a three-dimensional (3D) cross-ply laminate $[0 / 90]_{s}$. Both models were modelled in ABAQUS using 3D brick elements with element type C3D8. The temperature and degree of cure were considered as being known and therefore were defined as predefined field variables.

Rod Model. The rod was assumed to be $100 \mathrm{~mm}$ long with a cross section of $5 \mathrm{~mm} \times 5 \mathrm{~mm}$. Its axis was vertical to the fiber orientation. When the degree of cure is constant at $\alpha_{0}$ and the temperature history is given by $\Delta T h(t)$, where $h(t)$ is a unit step function, where $\Delta T=T_{1}-T_{0}$ is the temperature difference suddenly applied at $t=0$, then the exact solution for the axial stress $\sigma_{2}$ of the rod is obtained from Eq. (5)

$$
\sigma_{2}=\sum_{i=1}^{3}\left[C_{i i_{\infty}}+\sum_{m=1}^{M} C_{i i_{m}} e^{-t /\left(\rho_{m}\left(\alpha_{0}\right) a_{T}\left(\alpha_{0}, T_{1}\right)\right)}\right] \varepsilon_{i}
$$


where $\varepsilon_{2}=-\beta_{2} \Delta T, \varepsilon_{j}=-\varepsilon_{2} v_{2 j}(j=1$ and 3$)$.

The finite element model of the rod was 20 elements along its length and one element in the transverse section. The total calculation time was 330 minutes. Two groups of conditions ( $\alpha_{0}, T_{0}$ and $T_{1}$ ) were used to verify the relaxation behavior predicted by the proposed models at high temperature and room temperature respectively, Condition 1 assumed $\alpha_{0}=0.9, T_{0}=298 \mathrm{~K}$ and $T_{1}=398 \mathrm{~K}$ and Condition 2 assumed $\alpha_{0}=0.98, T_{0}=450 \mathrm{~K}$ and $T_{1}=298 \mathrm{~K}$. In order to examine the convergence of numerical solutions, the finite element analyses were performed for three time increments with $\Delta t=30$ seconds, 1 minute, and 2 minutes. There was no discernible difference between the predicted results using these time increments.

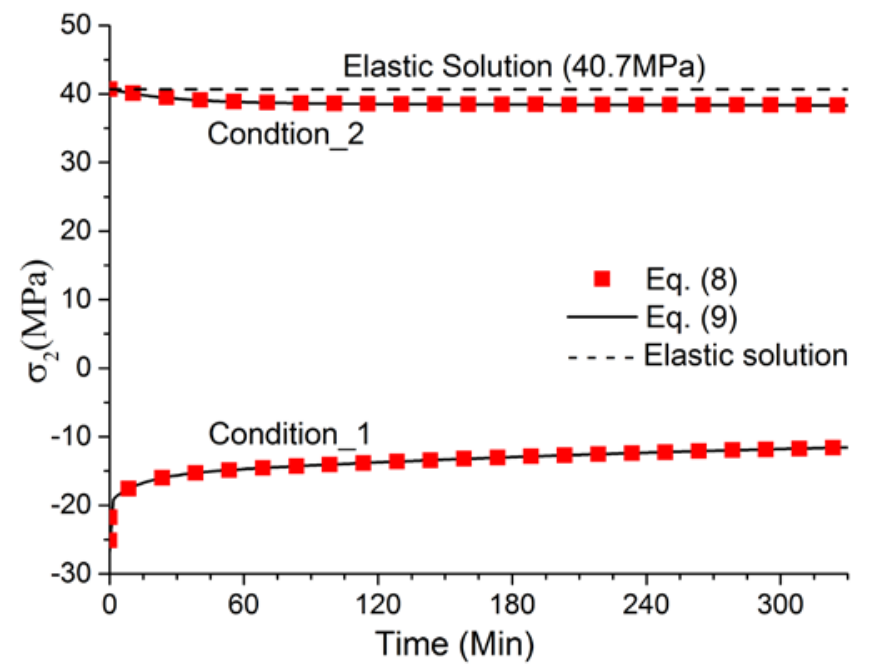

Figure 2. Comparison of the transverse stress $\sigma_{2}$ calculated by the exact solution and the proposed constitutive model for the rod under abrupt temperature change

The transverse stress $\sigma_{2}$ calculated by the exact solution Eq. (9) and the proposed model are compared in Fig. 2. They are in good consistent with each other. In different temperature and degree of cure condition, stress relaxation occurs during the whole calculation time, and it is more rapid in the initial stage. For the Condition 1 with high temperature, the stress relaxation is more serious. The elastic solution for condition 2 is also plot in Fig. 2 and it is $40.7 \mathrm{MPa}$. The exact solution at 330 minute is $38.3 \mathrm{MPa}$. There is a $6.3 \%$ relaxation of stress occurring in 330 minutes.
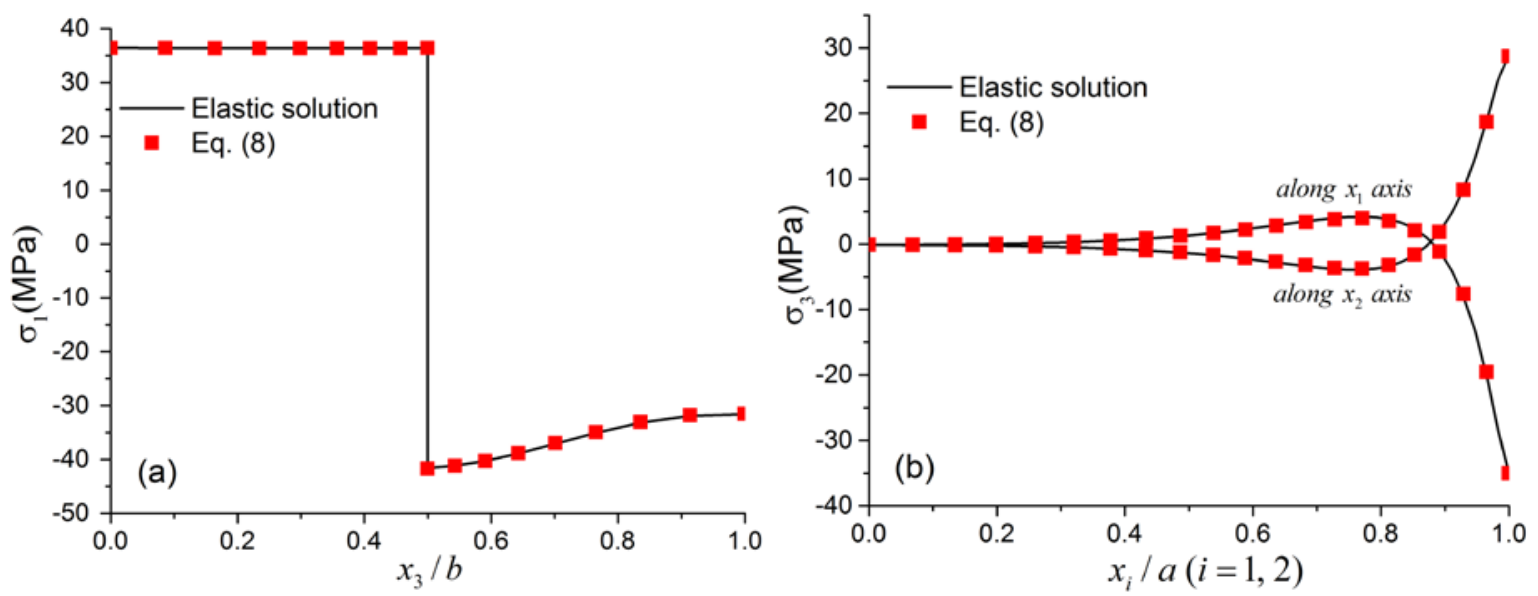

Figure 3. The distribution of the stress $\sigma_{1}$ at $x_{3}$ axis (a) and interlaminar normal stress $\sigma_{3}$ at $x_{1}$ and $x_{2}$ axes (b) for $\alpha_{0}=0.98$ with the abrupt temperature drop from $450 \mathrm{~K}$ to $298 \mathrm{~K}$. 
Plate model. Due to the symmetry of the geometry, only 1/8th of the model was modelled as shown in Fig. 1(c). 3200 elements were used, with 20 elements in the $x_{1}$ and $x_{2}$ directions and 8 in the $x_{3}$ direction. Elastic solutions predicted by the derived model were compared to those determined by using an ABAQUS elastic model. In order to produce the elastic solutions using the viscoelastic constitutive model, condition 2 mentioned in above was applied, and a very short total calculation time of $0.01 \mathrm{~s}$ was assumed.

The resulting interlaminar normal stresses $\sigma_{3}$ at $x_{1}$ and $x_{2}$ axes, and the stress $\sigma_{1}$ at $x_{3}$ axis are shown in Fig. 3. There is exact agreement between the standard ABAQUS elastic solution and the results obtained from the derived model. The maximum tensile transverse stress developed in the center of the plate is $36.4 \mathrm{MPa}$. Severe stress concentration occurs at the interface of the lateral surfaces as shown in figure 3(b), the maximum interlaminar normal stress $\sigma_{3}$ appears at $(0, a, 0)$ and it is $29 \mathrm{MPa}$. This may cause the interface debonding at the lateral surfaces after the cure process.

\section{Application to Composite Corner}

The constitutive model was used to investigate the effects of stress relaxation, chemical shrinkage and thermal contraction on the residual stresses and spring-in angle (as shown in Fig. 4(a)) created in the AS4/3501-6 composite corner during curing. The geometry model used in this work is shown in Fig. 4(b). The lay-up is $\left[45^{\circ} /-45^{\circ} / 0^{\circ} / 90^{\circ}\right] \mathrm{s}$. The direction along the length is taken as $90^{\circ}$, and the direction running from one arm to another is taken as $0^{\circ}$ as shown in Fig. 4(b). The thickness of each layer is $1 \mathrm{~mm}$ with a total nominal thickness of $8 \mathrm{~mm}$.
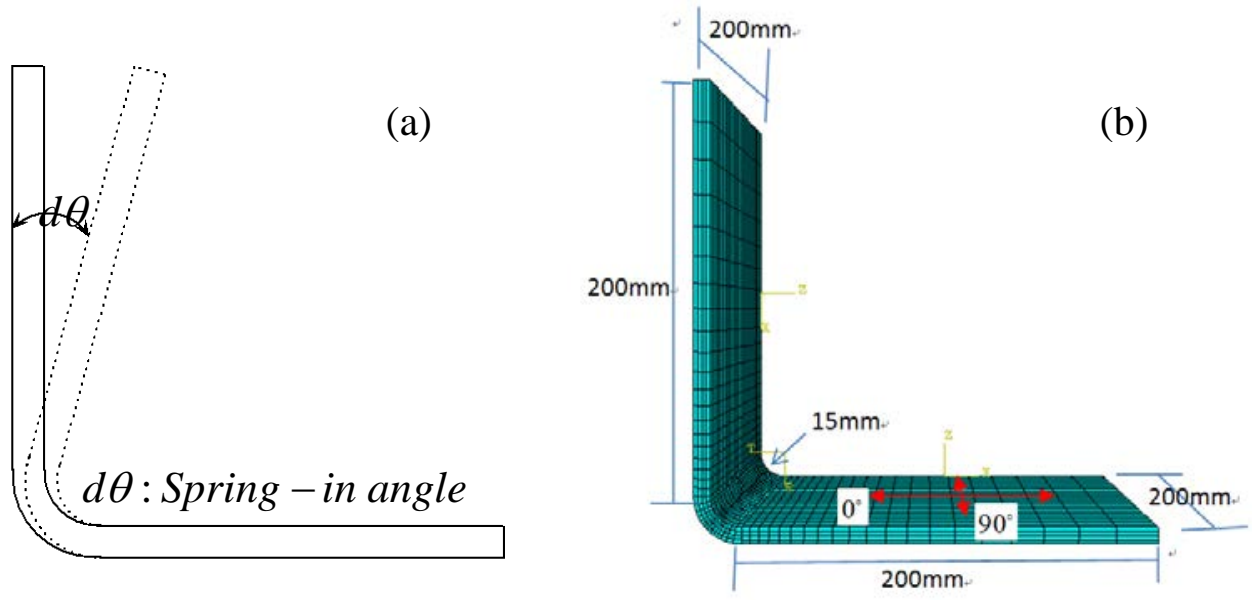

Figure 4. (a) Schematic of spring-in angle and (b) Geometry dimension of the composite corner

Fig. 5(a) shows the temperature cycle and the corresponding degree of cure history. The temperature of the first dwell is $389 \mathrm{~K}$ for one hour. The second dwell is $450 \mathrm{~K}$ for 2 hours. The rate of $2.5 \mathrm{~K} / \mathrm{min}$ was equally applied to the increase and the decrease of the temperature of the temperature cycle. With this temperature cycle, degree of cure was calculated on the basis of the cure kinetic equations for AS4/3501-6 composite in the references [16, 17]. It was assumed that the temperature and degree of cure were changed uniformly through the composite corner during curing.

To investigate the influences of different mechanical boundary conditions on the residual stress and spring-in predictions, two different mechanical boundary conditions were used. In the first case the composite corner was free to deform during the entire cure process with nodes constrained to suppress rigid body motion. In the second case the corner is considered to be bonded to an absolutely rigid convex mould, thus all nodes in the inner surface were fully constrained during the entire cure process. Then removing constraints so that only rigid body motion is suppressed 
simulated demoulding. Here only the composite corner was modelled. In reality, the constraints from the mould can be expected to be between these two extremes.
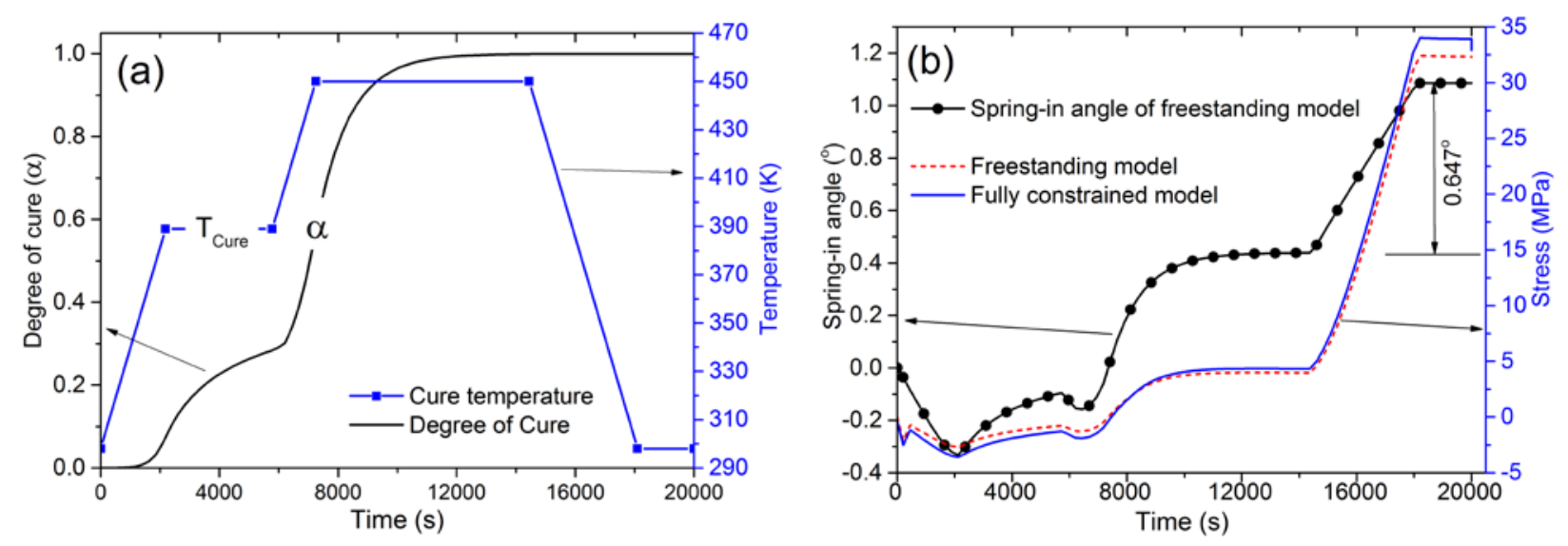

Figure 5. (a) Cure cycle applied to AS4/3501-6 composite corner and the predicted degree of cure history, and (b) the predicted spring-in angle for the freestanding model and the hoop stress in the center of the fillet corner

The predicted spring-in angle for the freestanding model and the hoop stress in the center of the fillet corner are shown in Fig. 5(b). Before the cooling process, the maximum hoop stress was 3.99 $\mathrm{MPa}$ and $4.68 \mathrm{MPa}$ at 208 minute for the freestanding and fully constrained model respectively. For the remainder of the second dwell, the residual stresses predicted by the viscoelastic models relaxed very slightly to $3.96 \mathrm{MPa}$ and $4.66 \mathrm{MPa}$ at 240 minute respectively. In reality, the stress relaxation was partly compensated by small amounts of chemical cure shrinkage during this period with the degree of cure changed from 0.996 at 208 minute to 0.999 at 240 minute. At the start of the cooling process, the predicted residual stress exhibited significant nonlinearity due to rapid stress relaxation as shown in Fig. 5(b).

For the freestanding model, spring-in developed during the entire cure process. There was an isothermal increase in spring-in angle at the dwell temperatures due to chemical shrinkage, and the spring-in angle attains $0.439^{\circ}$ just before cooling process. This is followed by a rapid increase in spring-in angle during cooling to room temperature after complete cure. The total spring-in angle is $1.086^{\circ}$ with $0.647^{\circ}$ produced during cooling process. This indicates that the spring-in caused by the chemical shrinkage during curing is significant. A standard elastic solution was also performed for the composite corner cooling from the cure temperature to room temperature with a freestanding constraint, and a spring-in angle of $0.674^{\circ}$ was obtained. It is higher than that calculated by the viscoelastic model for the cooling process. Thus the stress relaxation during the cooling process leads to a reduction in spring-in angle.

For the fully constrained model, as shown in Fig. 5(b), the predicted residual stresses are elevated instead of spring-in during the cure process. The residual stress predicted by the fully constrained model are higher than that predicted by the freestanding model. The residual stress was released at demoulding and spring-in angle formed. The hoop stress in the model center was released from 33.24MPa to $32.24 \mathrm{MPa}$ at demoulding, and the spring-in angle $0.986^{\circ}$ was produced. It is less than that of the freestanding model, whereas the final residual stress is higher than that of the freestanding model. The reason for the difference is that the deformation is frozen into the specimen due to the fully constrained boundary condition[5].

Fig.6 compares the hoop stress distribution along the thickness direction at the center of the fillet corner predicted by different models. The elastic model only concerning the cooling process underestimates the residual stress since the chemical shrinkage during the curing process is not considered. The residual stresses predicted by the fully constrained model are generally higher than those by the freestanding model. 


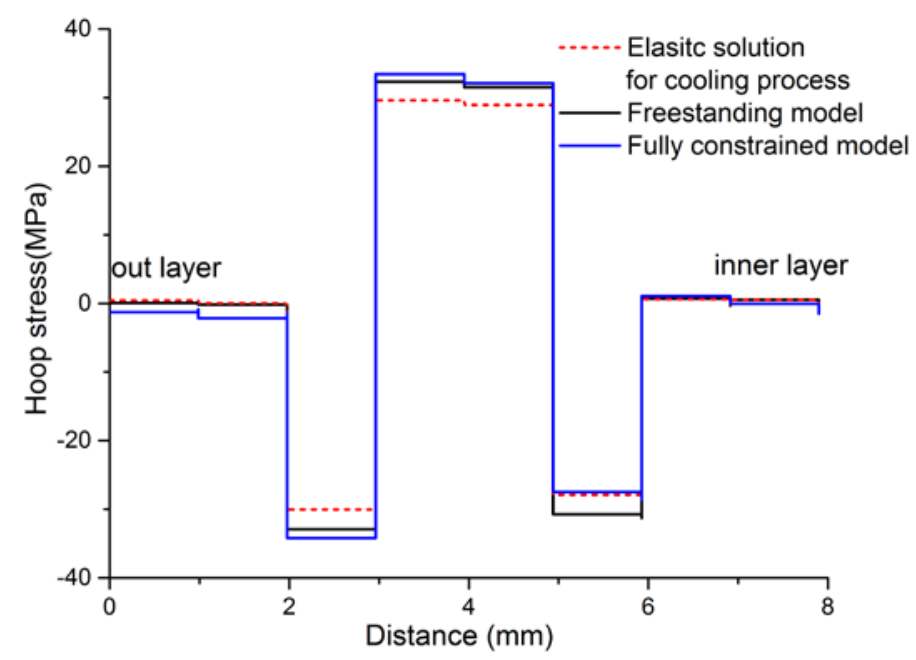

Figure 6. Hoop stress distribution along thickness direction at the center of the fillet corner

\section{Conclusions}

A cure-dependent viscoelastic constitutive model for PMCs during curing was simply derived, and verified by comparing their predictions with the theoretic solution of a rod model and the standard ABAQUS elastic solution of a three-dimensional (3D) cross-ply laminate [0/90]s. The constitutive model was then applied to investigate the effects of stress relaxation, chemical shrinkage and thermal contraction on the development of residual stresses and spring-in angle created in an AS4/3501-6 composite corner during curing, and the freestanding and fully constrained boundary conditions were used to study the influences of boundary condition on the predictions.

The results reveal that the spring-in caused by the chemical shrinkage during curing is significant and the stress relaxation during cooling process can lead to the reduction in spring-in angle. The residual stresses predicted by the freestanding model are less than those predicted by the fully constrained model, whereas the predicted spring-in angle is the inverse. The reason for the difference is that the deformation is frozen into the specimen due to the fully constrained boundary condition. The elastic model only concerning the cooling process underestimates the residual stress and spring-in angle since the chemical shrinkage during curing process is not considered. In summary, the model for the residual stresses and distortion prediction in composite component should include both the curing process and cooling process. Stress relaxation of the composite should be considered, and a proper boundary condition is important for the accuracy of the model predictions.

\section{Acknowledgements}

This work was supported by the National Natural Science Foundation of China (NSFC) under Grant No.11202154, and by the Fundamental Research Funds for the Central Universities of China under Grant No. 2016IB002.

\section{References}

[1] H.T. Hahn, N.J. Pagano, Curing stress in composite laminates, J. Compos. Mater. 9 (1975) 91-105.

[2] R.J. Stango, S.S. Wang, Process-induced residual thermal stresses in advanced fiber-reinforced composite laminates, J. Manuf. Sci. Eng. 10 (1984) 48-54.

[3] S.R. White, Y.K. Kim, Process-induced residual stress analysis of AS4/3501-6 composite material, Mech. Compos. Mater. Struct. 5 (1998) 153-186. 
[4] J.M. Svanberga, J.A. Holmberg, Prediction of shape distortions Part I. FE-implementation of a path dependent constitutive model, Compos. Part A 35 (2004) 711-721.

[5] J.M. Svanberga, J.A. Holmberg, Prediction of shape distortions. Part II. Experimental validation and analysis of boundary conditions, Compos. Part A 35 (2004) 723-734.

[6] T.A. Bogetti, J.W. Gillespie Jr, Process-induced stress and deformation in thick-section thermoset composite laminates, J. Compos. Mater. 26 (1992) 626-660.

[7] A. Johnston, R. Vaziri, A plane strain model for process-induced deformation of laminated composite structures, J. Compos. Mater. 35 (2001) 1435-69.

[8] M. Abouhamzeh, J. Sinke, K.M.B. Jansen, R. Benedictus, Closed form expression for residual stresses and warpage during cure of composite laminates, Compos. Struct. 133 (2015) 902 - 910.

[9] E. Ruiz, F. Trochu, Thermomechanical properties during cure of glass-polyester RTM composites: Elastic and viscoelastic modeling, J. Compos. Mater. 39 (2005) 881-915.

[10] D.J. O’brien, P.T. Mather, Viscoelastic properties of an epoxy resin during cure, J. Compos. Mater. 35 (2001) 883-904.

[11] S.R. White, H.T. Hahn, Process modeling of composite materials: residual stress development during cure. Part I Model Formulation, J. Compos. Mater. 26 (1992) 2402-22.

[12] S.R. White, H.T. Hahn, Process modeling of composite materials: residual stress development during cure. Part II. Experimental validation, J. Compos. Mater. 26 (1992) 2423-53.

[13] S.Y. Lee, J.K. Kang, Residual Stresses in a Laminated Shell During Cure, KSME Int. J. 13 (1999) 625- 633.

[14] C. Brauner, S. Bauer, A.S. Herrmann, Analysing process-induced deformation and stresses using a simulated manufacturing process for composite multispar flaps, J. Compos. Mater. 49 (2014) 387-402.

[15] J. Li, X.F. Yao, Y.H. Liu, Z.Z. Cen, Z.J. Kou, X.C. Hu, D. Dai, Thermo-viscoelastic analysis of the integrated T-shaped composite structures, Compos. Sci. Technol. 70 (2010) 1497-1503.

[16] M. Abouhamzeh, J. Sinke, K.M.B. Jansen, R. Benedictus, A new procedure for thermo-viscoelastic modelling of composites with general orthotropy and geometry, Compos. Struct. 133 (2015) 871-877

[17] A. Ding, S. Li, J Sun, J Wang, L Zu, A thermo-viscoelastic model of process-induced residual stresses in composite structures with considering thermal dependence, Compos. Struct. 136 (2016) $34-43$

[18] A. Ding, S. Li, J Sun, J Wang, L Zu, A comparison of process-induced residual stresses and distortions in composite structures with different constitutive laws, J. Reinf. Plast. Comp. 0(2016) $1-17$

[19] R.L. Taylor, K.S. Pister, G.L. Goudreau, Thermomechanical analysis of viscoelastic solids, Int. J. Numer. Meth. Eng. 2 (1970) 45-59.

[20] Y.K. Kim, S.R. White, Viscoelastic analysis of processing-induced residual stresses in thick composite laminates, Mech. Compos. Mater. Struct. 4 (1997) 361-387.

[21] Y.K. Kim, S.R. White, Process-induced stress relaxation analysis of AS4/3501-6 laminate, J. Reinf. Plast. Comp. 16 (1997) 2-16. 